\title{
A unique device enabling electrohydraulic lithotripsy with an ultraslim scope for difficult stones after endoscopic ultrasound-guided biliary drainage
}

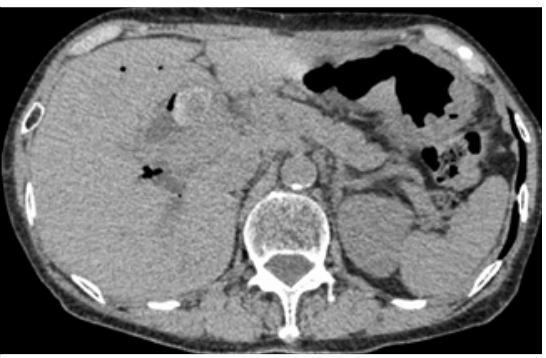

- Fig. 1 Abdominal computed tomography image showing the large common bile duct stone.

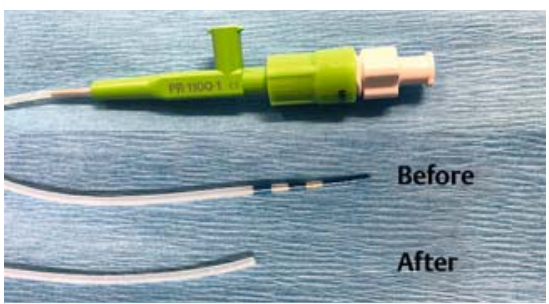

- Fig. 2 Photograph showing a tapered endoscopic retrograde cholangiopancreatography cannula that is reconfigured by cutting off its distal tapered end.
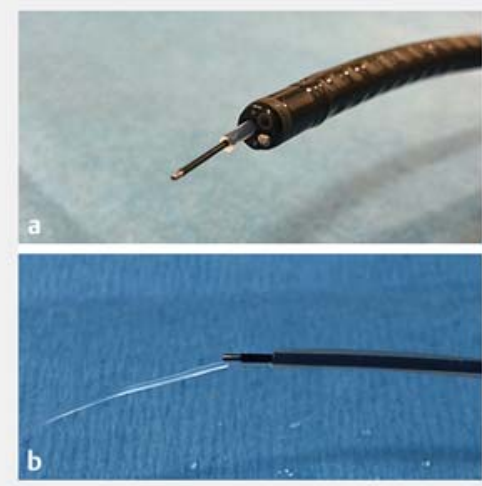

Fig. 3 Photographs showing: a the conventional ultraslim endoscope with the endoscopic retrograde cholangiopancreatography cannula emerging from the working channel, which allows the electrohydraulic lithotripsy (EHL) fiber to be passed through it; $\mathbf{b}$ manual irrigation to maintain the required fluid-based medium for effective EHL.
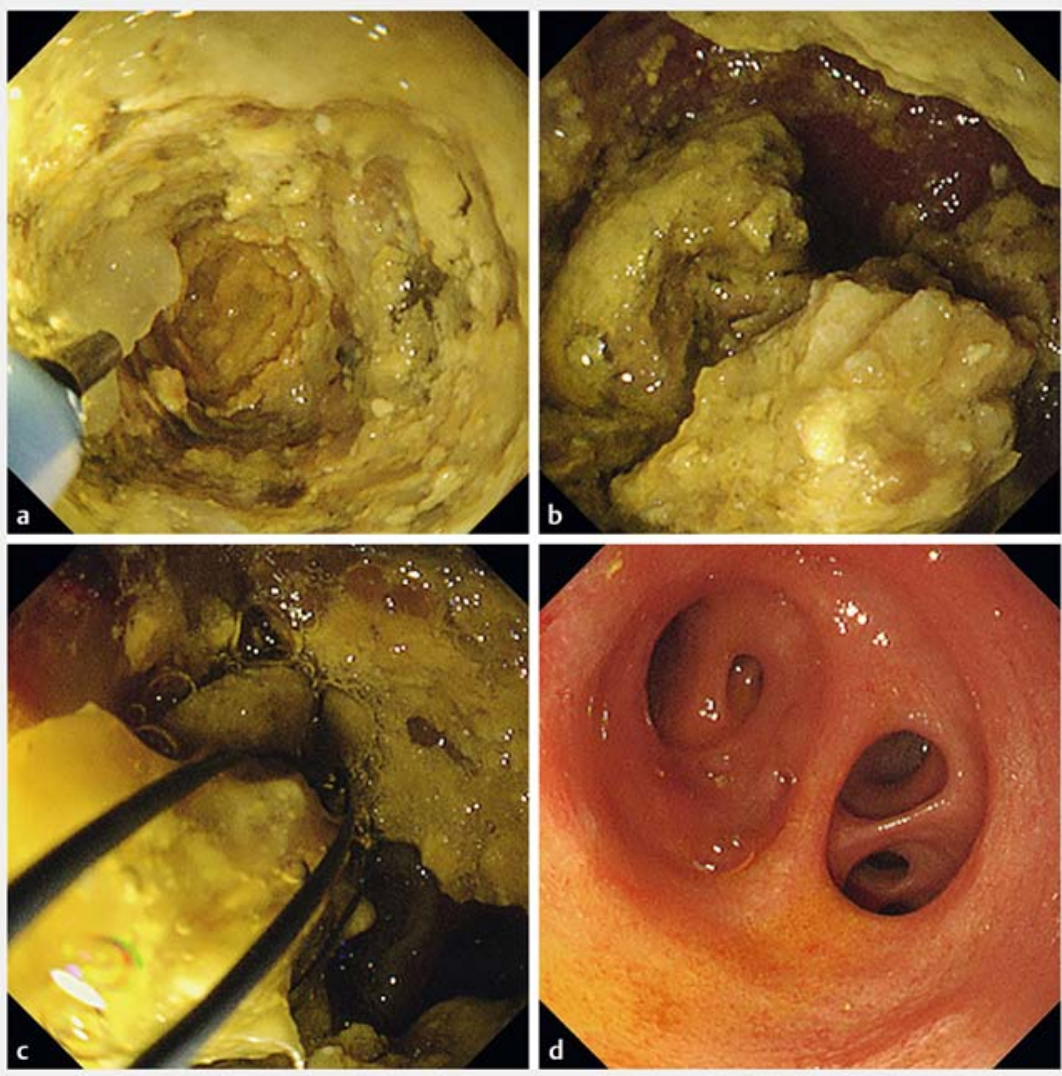

Fig. 4 Endoscopic images showing: a tunneling after EHL sparks were generated against the large stone stuck in the bile duct; $\mathbf{b}$ further fragmentation of the stone; $\mathbf{c}$ basket extraction of the remnant fragments; $\mathbf{d}$ the cleared bile duct after successful removal of the stone fragments.

Endoscopic ultrasound-guided biliary drainage (EUS-BD) is a well-established treatment option for benign biliary diseases after failure of conventional procedures [1]. A two-step approach of cholangioscopy-assisted management for a difficult biliary stone through an EUSguided bilioenteric fistula has previously been reported [2]. Cholangioscopyguided electrohydraulic lithotripsy (EHL) is currently considered one of the effective methods for stone removal, with clearance rates of $69 \%$ and $97 \%$ in single and multiple sessions, respectively [3]. Nevertheless, the high cost of digital cholangioscopy has limited its use in many centers [4]. Herein, we present a modified device that enabled EHL to be performed using a conventional ultraslim endoscope for a difficult biliary stone after EUS-BD.

The patient was an 80-year-old woman who presented with fever and jaundice. Her past history was significant for a gastroduodenostomy after gastric outlet obstruction with severe duodenal stenosis complicating acute pancreatitis. An EUS-guided choledochoduodenostomy (CDS) was performed later (3 years prior to this presentation) after an attack of 


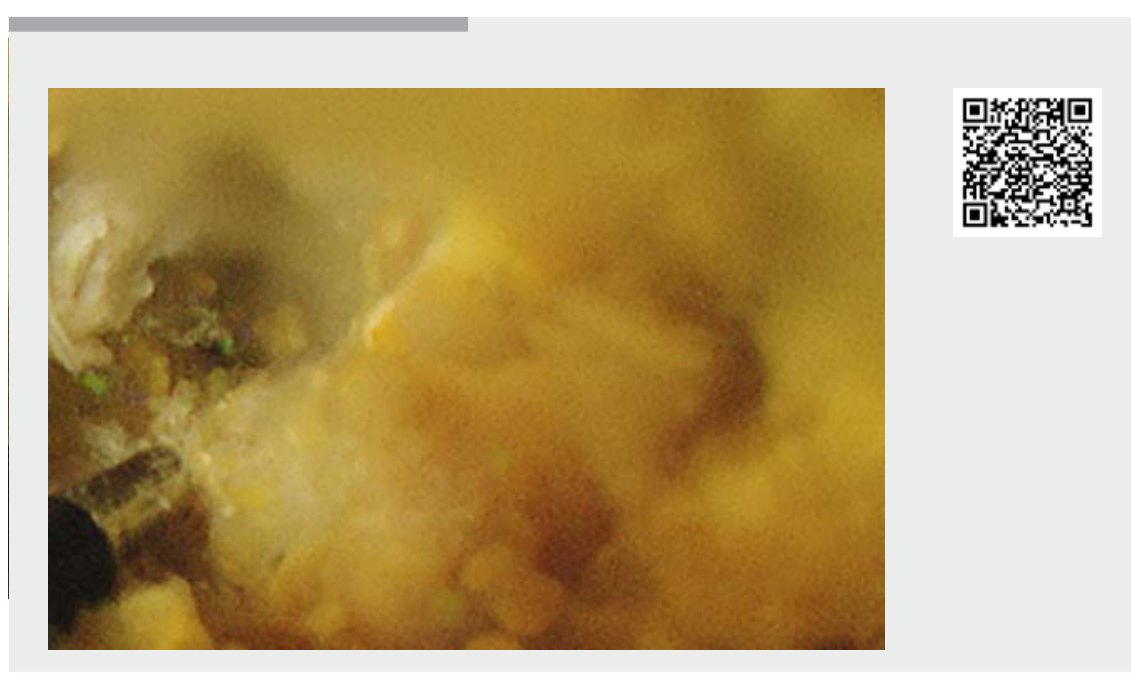

Video 1 A modified device is used to enable electrohydraulic lithotripsy with a conventional ultraslim endoscope passed through the fistula for a difficult common bile duct stone after previous endoscopic ultrasound-guided biliary drainage.

ascending cholangitis with the duodenal papilla inaccessible. An abdominal computed tomography (CT) scan revealed a large common bile duct (CBD) stone ( $\vee$ Fig. 1 ), but an initial trial of mechanical lithotripsy was unsuccessful in treating this.

We attempted intraductal EHL under direct visualization using an ultraslim scope (GIF-XP290N; Olympus) through the CDS fistula. A tapered endoscopic retrograde cholangiopancreatography (ERCP) cannula (PR-110Q-1; Olympus) was reconfigured by cutting off its distal tapered end ( $\triangleright$ Fig.2), which allowed the EHL fiber (EHL AUTOLITH TOUCH; Northgate Technologies Inc.) to be passed through the scope working channel and the required fluid-based medium for effective $\mathrm{EHL}$ to be maintained ( $\triangleright$ Fig.3). After saline irrigation of the CBD was achieved, EHL sparks were generated against the large stone that was stuck within the bile duct, leading to its tunneling and then further fragmentation ( $\mathbf{F i g . 4 a , b )}$. Thereafter, the stone fragments were successfully removed using a basket catheter ( $\triangleright$ Fig. $\mathbf{4}$ c, d; $\triangleright$ Video $\mathbf{1}$ ).

This modified device with its low cost and easy technique, without any complications, compared with digital singleoperator cholangioscopy represents a unique procedure that may be helpful for the treatment of difficult biliary stones after EUS-BD for benign indications.
[1] Nakai $\mathrm{Y}$, Kogure $\mathrm{H}$, Isayama $\mathrm{H}$ et al. Endoscopic ultrasound-guided biliary drainage for benign biliary diseases. Clin Endosc 2019; 52: 212-219

[2] Hosmer A, Abdelfatah MM, Law R et al. Endoscopic ultrasound-guided hepaticogastrostomy and antegrade clearance of biliary lithiasis in patients with surgically-altered anatomy. Endosc Int Open 2018; 6: E127E130

[3] Manes G, Paspatis G, Aabakken L et al. Endoscopic management of common bile duct stones: European Society of Gastrointestinal Endoscopy (ESGE) guideline. Endoscopy 2019; 51: 472-491

[4] Buxbaum JL, Abbas Fehmi SM. ASGE Standards of Practice Committee et al. ASGE guideline on the role of endoscopy in the evaluation and management of choledocholithiasis. Gastrointest Endosc 2019; 89: 1075-1105 e1015

\section{Bibliography}

Endoscopy_UCTN_Code_TTT_1AR_2AH

Endoscopy 2021; 53: E52-E53

DOI 10.1055/a-1180-7820

ISSN $0013-726 \mathrm{X}$

published online 5.6.2020

(c) 2020. Thieme. All rights reserved.

Georg Thieme Verlag KG, Rüdigerstraße 14

70469 Stuttgart, Germany

The authors declare that they have no conflict of interest.

\section{The authors}

Hassan Atalla ${ }^{1}$, Hideyuki Shiomi ${ }^{2}$, Takuya

Ikegawa ${ }^{2}$, Takashi Kobayashi ${ }^{2}$, Arata Sakai ${ }^{2}$, Atsuhiro Masuda ${ }^{2}$, Yuzo Kodama ${ }^{2}$

1 Hepatology and Gastroenterology Unit, Department of Internal Medicine, Mansoura Faculty of Medicine, Mansoura University, Mansoura, Egypt

2 Division of Gastroenterology, Department of Internal Medicine, Kobe University Graduate School of Medicine, Kobe, Japan

\section{Corresponding author}

\section{Hideyuki Shiomi, MD, PhD}

Division of Gastroenterology, Department of Internal Medicine, Kobe University Graduate School of Medicine, 7-5-1 Kusunoki-cho, Chuo-ku, Kobe, Hyogo, 650-0017, Japan Fax: +81-78-382-6309

hshiomi@med.kobe-u.ac.jp
This section has its own submission website at https://mc.manuscriptcentral.com/e-videos 